\title{
Mixotrophs: Dynamic Disrupters of Plankton Systems?
}

Irene M. Moroz* moroz@maths.ox.ac.uk

Mathematical Institute, University of Oxford, Andrew Wiles Building, ROQ, Oxford OX2 6GG, UK, Roger Cropp, Faculty of Environmental Sciences, Griffith University, Nathan, Queensland, Australia, John Norbury, Mathematical Institute, University of Oxford, Andrew Wiles Building, ROQ, Oxford OX2 6GG, UK

*Corresponding author.

January 25, 2019 


\begin{abstract}
We consider a simple plankton model with one phytoplankton autotroph population, one mixotrophic phytoplankton population that both photosynthesises and consumes the other phytoplankton, and one zooplankton predator population. The zooplankton predator may be either a specialist or a generalist grazer on the phytoplankton. We examine the influence on the system's equilibrium points and dynamical properties of varying degrees of mixotrophy by the phytoplankton and grazing strategy by the zooplankton, both with and without the presence of seasonal forcing.

We find that the strength of the mixotrophy interaction does not substantially change the equilibrium properties of the model across all grazing strategies, but it can have a substantial effect on the dynamical properties. Further, external forcings on typical phytoplankton or zooplankton time scales can in some circumstances substantially modify these dynamics. Our analyses suggest that plankton models developed to represent bio-geochemical processes in the ocean for applications in climate change modelling should be subjected to thorough equilibrium and dynamical analyses before being used for climate prediction.
\end{abstract}




\section{Introduction}

There is increasing evidence that the majority of plankton in the oceans are neither strictly autotrophs (i.e. photosynthesising phytoplankton) or strictly heterotrophs (i.e. phagotrophic zooplankton) but are mixotrophs (i.e. phytoplankton that also ingest other organisms, or zooplankton that also utilise photosynthetic products) [11]. This suggests that the classic work-horse model of bio-geochemical oceanography, the NPZ ( nutrient-phytoplankton-zooplankton) model [12], may need to be replaced with an NPMZ (nutrient-phytoplankton-mixotroph-zooplankton) model.

One of the earliest mathematical analyses of the influence of mixotrophy on plankton dynamics was by Thingstad et al [27] who examined an NBAMP (nutrient-bacteria-autotroph-mixotroph-phagotroph) chemostat model and concluded that there was no strategy available to the mixotroph that would allow a stable point where all four populations coexisted. Crane and Grover [6] modelled interacting populations with four nutritional strategies; heterotrophic bacteria, heterotrophic zooflagellates, phototrophic algae, and mixotrophs that photosynthesised and consumed bacteria. They concluded that mixotrophs expressing a wide range of mixotrophic strategies persisted in low-nutrient habitats, but only those with a strategy that was mostly autotrophic persisted with high-nutrient habitats, and then only if sufficient light was available. However, they predicted that organisms representing all four nutritional strategies they examined could coexist in habitats with high nutrient and light supplies. Mitra et al [21] showed in a numerical steady state analysis of a multi-nutrient, variable stoichiometry model with six plankton functional types that mixotrophs could potentially change the biological carbon pump in the oceans, with potentially profound implications for global climate change. This view is supported more generally by Ullah et al [28] who suggest that climate change could lead to the collapse of present-day plankton based marine ecosystems to simpler detritus-based systems. This suggests that understanding the role of mixotrophy in marine plankton ecosystems, and how the dynamics of those ecosystems will respond to climate change, may play a fundamental role in understanding and predicting the impacts of anthropogenic (in particular, carbon dioxide related) environmental change.

It is well-known that mixotrophic plankton can change their mixotrophic strategy in response to changes in their external environment, for example temperature increase [31]. Although the physiology of mixotrophs can be extremely complex [10] and their strategies dynamic [2], the influence of their various strategies on the population dynamics of the ecosystems in which they play a key role [27, 6, 21] may be approximated with the usual saturating (nonlinear) interaction terms. 
Thingstad et al [27] noted that the examination of equilibria of idealized models was a necessary preliminary to developing a deeper understanding of the dynamic behaviour of more complex/realistic models. Here, we examine the equilibria and dynamical properties of a simple NPMZ model to reveal the influence of mixotrophic interactions in models with different assumptions about the zooplankton grazing strategy. We also investigate how exogenous (seasonal or longer time-scale) forcings may interact with the endogenous dynamics of the ecosystem to modify the internal system dynamics in the various cases outlined above.

Previously we studied the dynamics of an ecosystem made up of two phytoplankton prey populations, $P_{1}, P_{2}$, and a predator zooplankton, $Z$, population in the presence of a limiting nutrient $N$ [8], [23]. In those papers, both phytoplankton populations were assumed to be autotrophs: each produced complex organic components via photosynthesis or from inorganic components. We also considered specialist grazing, where the predator feeds on multiple prey sources, independently of one another, and generalist grazing, where the predator feeds on a combination of both prey sources.

Here, we investigate a similar ecosystem model structure, but comprising a phytoplankton population $P$, a mixotroph population, $M$, and a zooplankton population, $Z$ in the presence of a limiting nutrient $N$. We consider the cases of both specialist and generalist grazing for $Z$, and compare the outcomes with our previous investigations. Of particular interest is whether the previous instances of regular or irregular system behaviour persists under longer time-scale seasonal forcings, appropriate to the IPCC studies.

In $\S 2$ we describe in detail the Nutrient-Phytoplankton-Mixotroph-Zooplankton (NPMZ) model under investigation, together with a table of typical parameter values. $\S 3$ contains a summary of the linear stability analysis for the various critical points, culminating in a two-parameter stability diagram, showing the regimes of existence for the key critical points and the associated regular system behaviour. In $\S 4$ we describe our numerical integrations for the unforced system for both a specialist and generalist predator. In particular, we investigate how the effects of mixotrophy influence the ecosystem, by producing bifurcation transition diagrams as the mixotrophy parameter varies. We include individual time series, which illustrate 'pinball dynamics', a phenomenon where the autotroph (phytoplankton) and mixotroph populations alternate in dominance, with the zooplankton tracking the autotroph. Irregular (chaotic) dynamics also occurs for certain parameter regimes. 
In $\S 5$ we introduce seasonal forcing on both phytoplankton and zooplankton time scales, and study the consequences of such forcings on the system for various mixotrophy-grazing scenarios. $§ 6$ contains the discussion, where we point out that user choices in model construction can significantly alter the outputs of interest in the model.

\section{The Plankton model}

The simple food web, given in Figure 1, shows the flow of limiting nutrient between plankton populations $P$ (the autotroph), $M$ (the mixotroph) and $Z$ (the predator), and the nutrient pool $N$. All populations and mass/nutrient flows are measured in terms of the limiting nutrient $N$.

We use Holling Type II nonlinearities, so that the dimensionless model equations, written in Kolmogorov form become:

$$
\begin{aligned}
\dot{P} & =P\left[\frac{\mu_{1} N}{N+\kappa_{1}}-\frac{\phi_{1} Z}{1+\epsilon_{1} P+\rho \epsilon_{2} M}-\frac{\phi_{3} M}{1+\epsilon_{3} P}-\sigma_{P}\right] \\
& =P F(P, M, Z), \\
\dot{M} & =M\left[\frac{\mu_{2} N}{N+\kappa_{2}}+\frac{\phi_{3}\left(1-\psi_{3}\right) P}{1+\epsilon_{3} P}-\frac{\phi_{2} Z}{1+\rho \epsilon_{1} P+\epsilon_{2} M}-\sigma_{M}\right] \\
= & M G(P, M, Z), \\
\dot{Z} & =Z\left[\frac{\phi_{1}\left(1-\psi_{1}\right) P}{1+\epsilon_{1} P+\rho \epsilon_{2} M}+\frac{\phi_{2}\left(1-\psi_{2}\right) M}{1+\rho \epsilon_{1} P+\epsilon_{2} M}-\sigma_{Z}\right] \\
& =Z H(P, M) .
\end{aligned}
$$

The choice $\rho=0$ means that $Z$ is a specialist omnivore, while $\rho=1$ means that $Z$ is a generalist omnivore. The populations are linked to the nutrient pool by limiting nutrient mass conservation on the food web when inputs from the external environment equal outputs to it:

$$
\dot{N}=-\dot{P}-\dot{M}-\dot{Z}
$$

so that $P+M+Z+N=N_{T}=1$. The Kolmogorov form for eq(2.1) with $d N / d t>0$ at $N=0$ ensures that all populations and the limiting nutrient in the nutrient pool are bounded between 0 and 1 , and so are ecologically sensible.

In addition, we call $Z$ a facultative grazer if it can survive on either $P$ or $M$ independently, and an obligate grazer if it requires either $P$ or $M$ to be present in order to survive.

Table 1 lists the various parameters appearing in eq(2.1), together with their interpretations, 
their reference sources and values. To facilitate comparison with the two autotroph study, we keep the same parameter values as in [23], apart from the additional parameter $\phi_{3}$.

Figure 1: Food web diagram for the $N P M Z$ model. Boxes show population sizes of $P, M, Z$ as well as the (abiotic) nutrient pool $N$, measured as a fraction of the total mass of limiting nutrient ( $N_{T}$ ). Arrows show flows of mass of limiting nutrient as fractions of the total per characteristic time, defined here as the inverse of the maximum $P$ growth rate $\left(\mu_{1}\right)$. Inflows from, and outflows to, the external environment of the limiting inorganic nutrient $N$ are generally an order of magnitude less than the recycling flows [29] and in this case are assumed to balance.

In this model $P$ is a simple autotroph that grows on the nutrient pool $N$ using solar energy to drive photosynthesis. The mixotroph $M$ can vary its trophic strategy between that of a simple autotroph $\phi_{3}=0$ or function as a mixotroph and additionally consume $P\left(\phi_{3}>0\right)$. The magnitude of $\phi_{3}$ determines the strength of this additional growth term in the model. A comparison between eq(2.1) and the corresponding model in [23] for the two prey populations reveals two additional terms that represent the mixotroph $M$ feeding on $P$ in addition to $N$ in eq(2.1a) and eq(2.1b).

The parameters listed in Table 1 , include an additional parameter, $\rho$, that specifies the grazing behaviour of $Z$. The extreme values of $\rho$ define $Z$ to be a purely specialist grazer ( $\rho=0$ ), or a purely generalist grazer $(\rho=1)$, while $0<\rho<1$ define mathematically valid, but ecologically less-intuitive, intermediate grazing strategies. Similarly, $\rho>1$, which is mathematically valid and is examined to "complete" the equilibrium analysis, has no generally accepted ecological interpretation. Note that when $M$ functions as a mixotroph $\left(\phi_{3}>0\right)$ it is always a specialist grazer, and the nominal per capita mixotrophic feeding rate is less than $10 \%$ of the comparable autotrophic rates.

Table 1: Measured parameter values for eqns eq(2.1), their physical interpretationsi and reference sources.

Par. Process Value Reference




\begin{tabular}{|c|c|c|c|}
\hline$\mu_{1}$ & Maximum (seasonal average) rate of $N$ uptake by & $1 P 00$ & Gabric et al. [13] \\
\hline$\mu_{2}$ & Maximum (seasonal average) rate of $N$ uptake by & $M 15$ & $\begin{array}{l}\text { Muller-Niklas and Hern، } \\
{[19]}\end{array}$ \\
\hline$\kappa_{1}$ & Half-saturation constant for $N$ uptake by $P$ & 0.25 & $\begin{array}{l}\text { Slagstad and Stole-Hans } \\
{[25]}\end{array}$ \\
\hline$\kappa_{2}$ & Half-saturation constant for $N$ uptake by $M$ & 0.07 & Billen and Becquevort [: \\
\hline$\phi_{1}$ & $Z$ grazing rate on $P$ & 6.18 & e et al. [16] \\
\hline$\phi_{2}$ & $Z$ grazing rate on $M$ & 1.85 & Gabric et al. [13] \\
\hline$\phi_{3}$ & $M$ grazing rate on $P$ & $0_{-} 00$ & \\
\hline $\mathrm{e}_{1}$ & Half-saturation constant for $Z$ uptake of $P_{1}$ & & Fenchel [9] \\
\hline$\epsilon_{2}$ & Half-saturation constant for $Z$ uptake of & & Fenchel [9] \\
\hline$\sigma_{1}$ & $P_{1}$ specific mortality rate & 0.00 & Gabric et al. [14] \\
\hline$\sigma_{2}$ & $P_{2}$ specific mortality rate & 0.26 & Moloney et al. [22] \\
\hline$\sigma_{Z}$ & $Z$ specific mortality rate & 0.19 & Moloney et al. [22] \\
\hline$\psi_{1}$ & Proportion of $P_{1}$ uptake excreted by $Z$ & 0.40 & Moloney et al. [22] \\
\hline$\psi_{2}$ & Proportion of $P_{2}$ uptake excreted by $Z$ & 0.40 & Moloney et al. [22] \\
\hline
\end{tabular}

\section{Critical Equilibrium Points}

The critical (or equilibrium) points are found by setting the LHS of eq(2.1) to zero, and are given in Table 2. Their linear stabilities are given by the Jacobian matrix, computed from eq(2.1) by linearising about each critical point. The Kolmogorov form of eq(2.1) provides an elegant representation of this matrix:

$$
J=\left(\begin{array}{ccc}
F+P F_{P} & P F_{M} & P F_{Z} \\
M G_{P} & G+M G_{M} & M G_{Z} \\
Z H_{P} & Z H_{M} & H+Z H_{Z}
\end{array}\right),
$$

where the critical points are the solutions to:

$$
P=0 \text { or } F=0, \quad M=0 \text { or } G=0, \quad Z=0 \text { or } H=0 .
$$

Since $H$ is independent of $Z$, the term $H_{Z}$ in eq(2.1)c is zero, which simplifies the subsequent 
linear stability analysis.

Table 2: The Critical points of eq(2.1) and their labels.

\begin{tabular}{lll}
\hline Critical Point & Label & $(P, M, Z, N)$ \\
\hline Origin & $\mathrm{O}$ & $(0,0,0,1)$ \\
Prey $P$ only & $\mathrm{A}$ & $(P, 0,0, N)$ \\
Prey $M$ only & $\mathrm{C}$ & $(0, M, 0, N)$ \\
Prey-Prey & $\mathrm{B}$ & $(P, M, 0, N)$ \\
Predator $Z$ only & $\mathrm{G}$ & $(0,0, Z, N)$ \\
Prey-Predator $(P, Z)$ & $\mathrm{D}$ & $(P, 0, Z, N)$ \\
Prey-Predator $(M, Z)$ & $\mathrm{F}$ & $(0, M, Z, N)$ \\
Prey-Prey-Predator & $\mathrm{E}$ & $(P, M, Z, N)$ \\
\hline
\end{tabular}

We now consider each critical point in turn. We use the $(P, M, Z)$-phase space for equations eq(2.1), where $N=1-P-M-Z$. However, in what follows, we include the value for $N$ as a reminder; so we show $(P, M, Z, N)$.

\subsection{The origin critical point $O$}

When $(P, M, Z, N)=(0,0,0,1)$, eq(3.2) usually has $(F, G, H) \neq(0,0,0)$ as the only non-zero entries in matrix $J$ in eq(3.1). Since these entries occur on the diagonal of $J$, they are the eigenvalues for the origin critical point. Moreover these eigenvalues (here $\lambda_{0 i}$ for $i=1,2,3$ ) are identical to those for the autotroph $\left(P_{1}, P_{2}, Z, N\right)$-model [23]:

$$
\lambda_{01}=\frac{\mu_{1}}{1+\kappa_{1}}-\sigma_{P}, \quad \lambda_{02}=\frac{\mu_{2}}{1+\kappa_{2}}-\sigma_{M}, \quad \lambda_{03}=-\sigma_{Z}
$$

The fact that the $P, M$ populations can grow on large supplies of $N$ makes both $\lambda_{01}$ and $\lambda_{02}$ positive; the origin is therefore an unstable saddle point.

\subsection{The autotroph prey $P$ only critical point $A$}

When $(P, M, Z, N)=(P, 0,0, N)$, the choice in eq(3.2) is $F=0$, so that 


$$
N=\frac{\sigma_{P} \kappa_{1}}{\mu_{1}-\sigma_{P}}=1-P
$$

with eigenvalues

$$
\lambda_{A 1}=-\frac{\mu_{1} \kappa_{1} P}{\left(N+\kappa_{1}\right)^{2}}, \quad \lambda_{A 2}=\frac{\mu_{2} N}{N+\kappa_{2}}+\frac{\phi_{3}\left(1-\psi_{3}\right) P}{1+\epsilon_{3} P}-\sigma_{M}, \quad \lambda_{A 3}=\frac{\phi_{1}\left(1-\psi_{1}\right) P}{1+\epsilon_{1} P}-\sigma_{Z} .
$$

$\mathrm{Eq}(3.4)$ and $\mathrm{eq}(3.5)$ are identical to the two autotroph model, apart from an extra term in $P$ in the equation for $\lambda_{A 2}$. Critical point $A$ can be either stable to unstable, depending on the signs of $\lambda_{A 2}$ and $\lambda_{A 3}$.

\subsection{The mixotroph prey $M$ only critical point $\mathrm{C}$}

When $(P, M, Z, N)=(0, M, 0, N)$, the choice in eq(3.2) is $G=0$, so that

$$
N=\frac{\sigma_{M} \kappa_{2}}{\mu_{2}-\sigma_{M}}, \quad=1-M
$$

with stability given by the eigenvalues

$$
\lambda_{C 1}=-\frac{\mu_{2} \kappa_{2} M}{\left(N+\kappa_{2}\right)^{2}}, \quad \lambda_{C 2}=\frac{\mu_{1} N}{N+\kappa_{1}}-\phi_{3} M-\sigma_{P}, \quad \lambda_{C 3}=\frac{\phi_{2}\left(1-\psi_{2}\right) M}{1+\epsilon_{2} M}-\sigma_{Z} .
$$

Here $\lambda_{C 1}$ and $\lambda_{C 3}$ correspond to $\lambda_{C 1}$ and $\lambda_{C 3}$ of [23]; $\lambda_{C 2}$ contains an extra term, due to mixotrophy. Again this critical point can be stable or unstable. We see that $\lambda_{C 1}<0$, while for $\phi_{3}=0$

, $\lambda_{C 2}>0$ for $\sigma_{P}=0$. Calculations show that $\lambda_{C 3}$ is positive for small values of $\sigma_{Z}$, and negative for larger values. The outcome is that when $\phi_{3}$ is sufficiently large and non-zero, $\lambda_{C 2}$ changes sign, thereby resulting in a stable prey only critical point $M$. For the parameter values shown in Table 1, this occurs when the mixotrophy parameter $\phi_{3} \geq 0.0772$.

\subsection{The prey-prey $P, M$ only critical point $B$}

When $\phi_{3}=0$ this critical point is a critical line, as in [23], and the dual prey critical point $(P, M, 0, N)$ only exists for certain combinations of the parameter values. However, when $\phi_{3}>0$ the critical line resolves to a critical point. The algebraic description of the location of this point involves the roots of two quadratics - this provides no insight into the location or properties of this point. Numerical analysis reveals that for the parameter values of Table 1 with $\rho=0$, it does not 
influence our equilibrium analysis because the third eigenvalue of this point $\left(\lambda_{3}=\left.H\right|_{B}\right)$ is always positive and the point is unstable for values of $\sigma_{z}$ up to 0.9 . However, when $\rho=1$ with Table 1 parameter values, this point is stable for $\sigma_{Z}>0.43$

Numerical evaluations also reveal that when the value of $\sigma_{Z}$ is large, the prey-prey point B is located near the boundary of the ecospace defined by $P+M=1$, as is often the case for autotroph/mixotroph critical points. This is supported by evaluation of the boundary critical points A and $\mathrm{C}$ that form the extreme values of the intersection of the zero isoclines $F=0=G$, which reveals that $\mathrm{A}$ is located at $\left\{P^{*}=1,0,0,0\right\}$ and $\mathrm{C}$ is located at $\left\{0, M^{*}=0.9796,0, N^{*}=0.0204\right\}$. In order for $Z$ to exist in this ecosystem, either all three prey-only boundary critical points $\mathrm{A}, \mathrm{B}$ and $\mathrm{C}$ must be unstable, or when $\mathrm{B}$ is stable (for $\sigma_{Z}>0.43$ when $\rho=1$ ) a separatrix must exist between the equilibrium points B and E. Figure 2 suggests that this is the case and the basin of attraction for B when it is stable is quite small and near the boundary of the ecospace defined by $P+M=1$. We are primarily interested in the properties of the system with all populations extant, and do not consider the properties of this point further.

\subsection{The predator $Z$ only critical point $G$}

The predator only critical point $(0,0, Z, N)(H \neq 0)$ does not exist unless $\sigma_{Z}=0$ (see eq(2.1c)), an entirely unrealistic scenario, which is not considered further.

\subsection{The prey-predator $(P, 0, Z, N)$ critical point $\mathbf{D}$}

The autotroph prey-predator critical point $(P, 0, Z, N)$ is given by:

$$
P=\frac{\sigma_{Z}}{\phi_{1}\left(1-\psi_{1}\right)-\epsilon_{1} \sigma_{Z}}, \quad Z=\left(\frac{1+\epsilon_{1} P}{\phi_{1}}\right)\left(\frac{\mu_{1} N}{N+\kappa_{1}}-\sigma_{P}\right) .
$$

Since we can replace $Z$ by $1-N-P$, eq(3.8) gives a quadratic equation for $N$, in terms of $P$ :

$$
d_{2} N^{2}+d_{1} N+d_{0}=0
$$

where

$$
\begin{gathered}
d_{2}=\phi_{1}, \\
d_{1}=\phi_{1}\left(P-1+\kappa_{1}\right)+\left(\mu_{1}-\sigma_{P}\right)\left(1+\varepsilon_{1} P\right),
\end{gathered}
$$




$$
d_{0}=-\sigma_{P} \kappa_{1}\left(1+\epsilon_{1} P\right)-\phi_{1} \kappa_{1}(1-P) .
$$

However its stability is found from

$$
\lambda_{D 1}=\frac{\mu_{2} N}{N+\kappa_{2}}+\frac{\phi_{3}\left(1-\psi_{3}\right) P}{1+\epsilon_{3} P}-\frac{\phi_{2} Z}{1+\rho \epsilon_{1} P}-\sigma_{M},
$$

and the roots of

$$
\lambda^{2}-\left(P F_{P}\right) \lambda-P Z\left(F_{Z} H_{P}\right)=0
$$

where $F_{P}, H_{P}$ are partial derivatives of $F$ and $H$ with respect to $P$ :

$$
\begin{gathered}
F_{P}=-\frac{\mu_{1} \kappa_{1}}{\left(N+\kappa_{1}\right)^{2}}+\frac{\phi_{1} \epsilon_{1} Z}{\left(1+\epsilon_{1} P\right)^{2}}, \\
F_{Z}=-\frac{\mu_{1} \kappa_{1}}{\left(N+\kappa_{1}\right)^{2}}-\frac{\phi_{1}}{1+\epsilon_{1} P}, \\
H_{P}=\frac{\phi_{1}\left(1-\psi_{1}\right)}{\left(1+\epsilon_{1} P\right)^{2}}
\end{gathered}
$$

Recall that $H_{Z}=0$. The mixotrophy term $\phi_{3}$ only appears in $G$, and so only in the real eigenvalue $\lambda_{1}$. The only bifurcation that can occur involving $(P, 0, Z, N)$ is a steady state bifurcation, in which $\lambda_{1}$ passes through zero; Hopf bifurcations are not influenced by mixotrophy and the expressions for the various derivatives of $F$ and $H$, appearing in eq(3.11) are in fact identical to their expressions for the autotroph case (see [23] eqn eq(3.12)). When Table 1 parameter values are used, it transpires that critical point $D$ can undergo a Hopf bifurcation when $\sigma_{Z} \approx 0.48$, as in the autotroph case. However this occurs when $\lambda_{1}$, given by eq(3.11), is positive, yielding a subcritical Hopf bifurcation to unstable limit cycles.

\subsection{The prey-predator $(0, M, Z, N)$ critical point $\mathbf{F}$}

When $P=0$, but neither $M$ nor $Z$ are zero, eq(3.2) gives $G=H=0$, so that:

$$
M=\frac{\sigma_{Z}}{\phi_{2}\left(1-\psi_{2}\right)-\epsilon_{2} \sigma_{Z}}, \quad Z=\left(\frac{1+\epsilon_{2} M}{\phi_{2}}\right)\left(\frac{\mu_{2} N}{N+\kappa_{2}}-\sigma_{M}\right) .
$$

Elimination of $Z$ via $Z=1-M-N$ gives

$$
f_{2} N^{2}+f_{1} N_{D}+f_{0}=0
$$

where 


$$
\begin{gathered}
f_{2}=\phi_{2}, \\
f_{1}=\phi_{2}\left(M-1+\kappa_{2}\right)+\left(\mu_{2}-\sigma_{Z}\right)\left(1+\epsilon_{2} M\right), \\
f_{0}=-\sigma_{M} \kappa_{2}\left(1+\epsilon_{2} M\right)-\phi_{2} \kappa_{2}(1-M) .
\end{gathered}
$$

Again mixotrophy means that an additional term appears in the eigenvalue equation for $\lambda_{1}$ :

$$
\lambda_{1}=\frac{\mu_{1} N}{N+\kappa_{1}}-\phi_{3} M-\frac{\phi_{1} Z}{1+\rho \epsilon_{2} M}-\sigma_{P},
$$

but not in the quadratic equation for the remaining two eigenvalues:

$$
\lambda^{2}-M G_{M} \lambda-M Z G_{Z} H_{M}=0 .
$$

The coefficients $G_{M}, G_{Z}, H_{M}$ are derivatives of $G$ and $H$, evaluated at the critical point:

$$
\begin{gathered}
G_{M}=-\frac{\mu_{2} \kappa_{2}}{\left(N+\kappa_{2}\right)^{2}}+\frac{\phi_{2} \epsilon_{2} Z}{\left(1+\epsilon_{2} M\right)^{2}}, \\
G_{Z}=-\frac{\mu_{2} \kappa_{2}}{\left(N+\kappa_{2}\right)^{2}}-\frac{\phi_{2}}{1+\epsilon_{2} M}, \\
H_{M}=\frac{\phi_{2}\left(1-\psi_{2}\right)}{\left(1+\epsilon_{2} M\right)^{2}} .
\end{gathered}
$$

We see that $G_{Z}<0$ and $H_{M}>0$, while $G_{M}$ can take either sign. As in the previous case, $\phi_{3}$ only arises in eq(3.17) for $\lambda_{1}$, while the equations for the various terms in eq(3.16) are identical to their forms in [23]. Thus critical point $F$ can undergo both a steady state and a Hopf bifurcation, just as for critical point $F$ in [23].

\subsection{The prey-prey-predator critical point $\mathrm{E}$}

We adopt the same procedure as in [23] to find the stability boundaries for the interior critical point $(P, M, Z, N)$. We fix $\phi_{3}=0.07$ and integrate system eq(2.1) and eq(2.2) numerically, noting when the maxima and minima values of $M$ in each oscillatory cycle coincide for given values of $\sigma_{z}$ and $\rho$. The values of $\sigma_{z}$ and $\rho$ are then varied $-\sigma_{z}$ between 0 and 0.6 , and $\rho$ between 0 (the specialist limit) and $\rho=1.3$ (beyond the generalist limit of $\rho=1$, in order to find where $E$ becomes a stable steady state). 


\subsection{Linear stability boundaries}

The above analysis reveals that only the locations of the critical points $\mathrm{B}\left\{P^{*}, M^{*}, 0\right\}$ and $\mathrm{E}$ $\left\{P^{*}, M^{*}, Z^{*}\right\}$ are sensitive to the value of the mixotrophy grazing parameter $\phi_{3}$. However, $\phi_{3}$ is involved in the stability properties of five critical points: A $\left\{P^{*}, 0,0\right\}, \mathrm{B}\left\{P^{*}, M^{*}, 0\right\}, \mathrm{C}\left\{0, M^{*}, 0\right\}$, $\mathrm{D}\left\{P^{*}, 0, Z^{*}\right\}$, and $\mathrm{E}\left\{P^{*}, M^{*}, Z^{*}\right\}$. Numerical analysis (see below) reveals that, for the parameter set in Table 1, the system collapses to just a $P$ population when $\phi_{3} \approx 0.08$. We therefore set $\phi_{3}=0.07$ to investigate the stability properties of the $N P M Z$ model to compare with the same analysis of the $N P_{1} P_{2} Z$ model in [23].

Figure 2 shows the linear stability boundaries in the $\left(\rho, \sigma_{z}\right)$-plane for critical points $(A, D, F)$, together with the nonlinear stability curves for critical point $E$ for $\phi_{3}=0.07$. We have also included for comparison the curve $S S D_{0}$ for the steady state bifurcation for critical point $D$ when $\phi_{3}=0$ (black) for the autotroph, shown as Figure 1 in [23].

Figure 2: Linear stability boundaries and regions of stability for the various critical points, computed using the parameter values of Table 1 , but with $\phi_{3}=0.07$. Here $S S n$ denotes a steady state bifurcation for critical point $n$ and $H B n$ denotes a Hopf bifurcation for critical point $n$. For comparison, we also include the stability boundary $S S D_{0}$ for the autotroph case.

The addition of a mutualism interaction of relatively high strength (around $90 \%$ of the value at which mixotrophy induces catastrophic collapse of the ecosystem) has very little effect on the stability properties of the plankton system, as Figure 2 above and Figure 1 of [23] are virtually identical. This rather counter-intuitive result suggests that while $\phi_{3}$ has only a small effect on the locations of the critical points, in particular the coexistence point $\mathrm{E}$, it has a much more significant effect on the stabilities of the critical points. This may be because the eigenvalues of the critical points are much more sensitive to $\phi_{3}$, or that the default parameter set produces eigenvalues that are delicately balanced between stable and unstable states. The numerical analysis below investigates these properties and how they influence the dynamics of the system, in particular for the generalist $\rho=1$ case. 


\section{$4 \quad$ Numerical Integrations}

Figures 3 and 4 show bifurcation transition diagrams for the maximum values of $P$ and $M$ respectively for $\sigma_{Z}=0.25$ as $\phi_{3}$ is increased from $\phi_{3}=0$. The upper panel in each plot corresponds to the specialist grazer case of $\rho=0$, while the lower plot corresponds to the generalist grazer case of $\rho=1$. In all cases, there was a loss of periodic behaviour when $\phi_{3}>0.0772$, the value at which critical point $C$ (the prey-only critical point $M$ ) undergoes a steady state bifurcation, leading to the loss of stability of the interior critical point $E$.

When compared to the previous study of two autotroph prey (where $\phi_{3}=0$ see [23]), the main difference is for the generalist grazer case (the lower panels in Figures 3 and 4). For very small values of $\phi_{3}$, the dynamics is simple and periodic, whereas for $\phi_{3}>0.04$, there is a transition to more complicated dynamics. This is further evident in the bifurcation transition diagrams as $\sigma_{z}$ varies. Figures 5 and 6 show a comparison of $P_{2}$ from [23] (left hand panel) with $M$ (right hand panel) for $\phi_{3}=0.07$. For the specialist case, shown in Figure 5, there is very little difference between the two bifurcation transition diagrams. Near the limit of $P_{2}=1$ and $M=1$, there are fewer maxima of the oscillations in the mixotroph case.

The picture is very different for the generalist plots of Figure 6. For the autotroph case, the left hand plot of Figure 6 shows very simple oscillatory or steady state solutions. This is in marked contrast to the mixotroph case shown as the right hand plot, where we see periodic, chaotic, period-halving bifurcations and windows of periodicity, as well as the simple dynamics for $\sigma_{z}<0.1$ . Clearly mixotrophy has complicated the dynamics. This is not inconsistent with Figure 2, since we did not indicate where critical point $E$ undergoes irregular or multi-periodic oscillations.

Figure 3: Bifurcation transition diagrams for the maximum value of $P$ over each oscillatory cycle as $\phi_{3}$ increases when $\sigma_{Z}=0.25$. The upper panel is the specialist grazer case of $\rho=0$, while the lower panel is the generalist grazer case of $\rho=1$.

Figure 4: As for Figure 2, but for the maxima of $M$ over each oscillation.

Figure 5: Bifurcation transition diagrams for the maximum values (blue) and minimum values (red) 
of (left panel) $P_{2}$ for the autotroph case from [23] and (right panel) $M$ over each oscillatory cycle as $\sigma_{Z}$ increases when $\rho=0$ and $\phi_{3}=0.07$.

Figure 6: Bifurcation transition diagrams for the maximum values (blue) and minimum values (red) of (left panel) $P_{2}$ for the autotroph case of [23] and (right panel) $M$ over each oscillatory cycle as $\sigma_{Z}$ increases when $\rho=1$ and $\phi_{3}=0.07$.

Figure 7: Time series of $P$ (blue), $M$ (black) and $Z$ (red) for (a) Specialist and (b) Generalist predator. Here $\sigma_{Z}=0.25$ and $\phi_{3}=0.07$. The remaining parameters are given in Table 1

Figure 7 shows a section of the time series for both the Specialist (a) and Generalist (b) grazer when $\sigma_{Z}=0.25$ and $\phi_{3}=0.07$. In case (a), the autotroph $P$ and the predator $Z$ vary together. During rapid oscillations, $P$ leads $Z$. When $P$ becomes very small, the mixotroph $M$ grows and becomes established over a long time frame, before declining, as $P$ takes over. The period of existence of each population is not regular. In case (b), the behaviour is more regular in all three populations, with much shorter periods for the dominance of $M$, before the growth of $P$ and $Z$. Both are examples of 'pinball dynamics', where orbits in the $(P, M, Z)$-phase space approach vertices $P=1$ or $M=1$ or $Z=1$ very closely, so that the vast majority of the available limiting nutrient in the system is taken up by one population in turn. The only difference is the time scale between growth and decay.

Comparison of Figure 5(b) here with Figure 2 of [23] suggests that the addition of mixotrophy has made very little difference to the dynamical properties of the system where the zooplankton functions as a pure specialist grazer $\left(\rho=0, \phi_{3}=0.07\right)$. However, comparison of Figure 6(b) with Figure 3 of [23] reveals that this is not the case for the generalist grazer $\left(\rho=1, \phi_{3}=0.07\right)$. Whereas the simple phytoplankton population in the latter case underwent a single Hopf bifurcation from a steady state to a limit cycle and back as $\sigma_{z}$ increased, the mixotroph enters a chaotic regime before eventually settling down to a period-one orbit, and then undergoing a Hopf bifurcation back to a steady state just prior to its eventual extinction. Figures 3 and 4 reveal that this chaotic regime extends to $P$, and is also sensitive to the value of $\phi_{3}$, with low level mixotrophy retaining the simple limit cycle dynamics of the $N P_{1} P_{2} Z$ system, but with chaotic dynamics developing by $\phi_{3} \approx 0.06$, about $75 \%$ of its useful range. 


\section{$5 \quad$ Seasonal forcing}

Because phytoplankton population explosions and declines are affected by seasonal variations in both sunlight and nutrients, it makes sense to investigate how such seasonal forcings affect the behaviours, discussed in the previous section. Following [23], we introduce forcings on the $\mu_{j}$ parameters, which represent the rate of uptake of nutrient $N$, and write:

$$
\mu_{j}(t)=(1+A \sin (\omega t)) \mu_{j}
$$

where $0 \leq A \leq 1$ is the amplitude of the forcing. The forcing frequency $\omega$ was chosen in two ways, one $\omega_{P}=2 \pi / 365$ based upon the phytoplankton time scale, and the second $\omega_{Z}=\omega_{P} / 9$ based upon the zooplankton time scale (so that 10,000 time units correspond to $\approx 3$ years).

We next compare the seasonally unforced Figure 4, with the seasonally forced scenario for both the specialist and generalist grazer when $\sigma_{Z}=0.25$. Figure 8 shows the behaviours with the phytoplankton forcing. The presence of seasonal forcing enables non-steady mixotroph dynamics to persist for much larger values of $\phi_{3}$. When $\rho=0$, the predator becomes extinct when $\phi_{3} \approx 0.174$, leaving a forced dual prey $(P, M, 0, N)$-state, before the autotroph $P$ becomes extinct when $\phi_{3} \approx 0.19$, leaving a periodic $(M, N)$-state. When $\rho=1$, we obtain the dual prey $(P, M . N)$-state when $\phi_{3} \approx 0.176$, and the periodic $(M, N)$-state when $\phi_{3} \approx 0.19$ as in the specialist case.

Figure 8: Bifurcation transition diagrams for the maximum values of $M(t)$ over each oscillatory cycle as $\phi_{3}$ increases for $\sigma_{Z}=0.25$ with seasonal forcing, based on the phytoplankton time scale. The upper panel is for $\rho=0$, while the lower panel is for $\rho=1$

In contrast Figure 9 shows the corresponding bifurcation transition diagrams when the zooplankton time scale is used for the seasonal forcing.

Figure 9: Bifurcation transition diagrams for the maximum values of $M(t)$ over each oscillatory cycle as $\phi_{3}$ increases for $\sigma_{Z}=0.25$ with seasonal forcing, based on the zooplankton time scale. The upper panel is for $\rho=0$, while the lower panel is for $\rho=1$ 
We next fix $\phi_{3}=0.07$ and vary $\sigma_{z}$, to obtain Figure 10, the seasonally forced analogue to Figure 6. For clarity, here we only plot maxima of $M$, rather than both the maxima and minima. This plot should be compared with Figure 6 of [23].

Figure 10: Bifurcation transition diagrams for the maximum values of $M(t)$ over each oscillatory cycle as $\sigma_{Z}$ increases and decreases for $\rho=1$ and $\phi_{3}=0.07$ with seasonal forcing, based on (a) the zooplankton time scale and (b) the phytoplankton time scale.

[[Image]]

Figure 11: Sections of time series for $P$ (blue), $M$ (black) and $Z$ (red) for (a) the Specialist and (b) Generalist grazer, under seasonal forcing, based on the predator timescale $\omega_{z}$. Here $\sigma_{Z}=0.25$ and $\phi_{3}=0.07$.

Figure 11 shows the effects of seasonal forcing on the zooplankon time scale when $\sigma_{Z}=0.25$ and $\phi_{3}=0.07$. (We also investigated the corresponding situation with phytoplankton timescales, and found the dynamics to be more regular.) This figure should be contrasted with Figure 7 (the unforced case). We again find $P$ and $Z$ evolving together, but now the amplitude of $P$ decreases as that of $Z$ increases. When both become very small, $M$ takes over. However, it is possible to find sections of the time series (see 11(b) for $t \approx 17500-18000$ ), where all three populations co-exist, with $M$ evolving out of phase with both $P$ and $Z$. Again $P$ and $Z$ oscillate more rapidly than $M$.

\section{Discussion}

Comparison of Figure 2 of this manuscript with Figure 1 of Moroz et al [23] suggests that the transition of $P_{2}$ from a pure autotroph in the $N P_{1} P_{2} Z$ model of [23] to a mixotroph in the NPMZ model has had very little impact on the equilibrium properties of the model, as these two figures appear nearly identical. This is supported by comparison of Figure 2 in [23] with Figure 5(b) of this manuscript (the specialist $Z$ case), which again are very similar if not identical. However, a difference is evident when comparing Figure 3 of [23] with Figure 6(b) of this manuscript (the 
generalist $Z$ case). Here, the simple limit cycle dynamics of the autotroph are replaced by irregular (chaotic) dynamics of the mixotroph. As the generalist case of Figure 6 is commonly used in Dynamic Green Ocean models being developed for climate simulations (DGOMs, [1, 4, 24]) this could have significant implications for predictions of the ocean biological carbon pump [21]. If these models were to include mixotrophy, as has been suggested by a number of authors [11, 21], it could have profound implications for their predictions of future climate. Figures 3 and 4 suggest that specialist mixotrophy is inherently unstable, except at very large mixotrophic grazing pressure. However, large values of $\phi_{3}$ that stablise the system are only marginally smaller than values that cause the ecosystem to collapse.

Our results suggest that changes in the dynamics of the biological carbon pump could be extremely difficult to detect or predict. The equilibrium analysis of $\S 3$ and Figures 2, 5 and 6 show the inclusion of mixotrophy at moderate levels, $\phi_{3} \approx 0.07$. Recall from $\S 4$ that when $\phi_{3} \approx 0.0772$ both $P$ and $Z$ go extinct leaving only an $N M$ system, Figures 2, 5 and 6 show the effect of the mixotroph when it "dominates" the system in the context that if its mixotrophic behaviour increases by around $10 \%$ it drives all other populations to extinction. This suggests that steady-state analyses of the effect of allowing a phytoplankton population to become mixotrophic on the properties of the system may underestimate the potential impact, and we should heed the advice of Thingstad [27] that steady-state analyses are an important step, but in fact a necessary prelude to an investigation of the dynamical properties of the models. These results suggest that extensive numerical investigations of the dynamics of ecosystem models involving mixotrophy may be fundamental to understanding their properties and how these properties influence the predictions they make of how plankton systems will respond to environmental change.

Figure 7(b) in particular suggests that the PMZN system may exhibit 'pinball dynamics' ([7, 20] where the eigenvectors of multiple critical points closely control the dynamics. This is evidenced by the autotroph $P$ and predator $Z$ populations closely interacting in bursts of rapid oscillations, while the mixotroph $M$ is very small, for long periods of time. Then $M$ is re-established, as $P$ and $Z$ become small until the $P Z$ oscillations once again take over. Detailed understanding of the dynamics of these systems requires the location and tracking of all the critical points and their eigenvectors, a substantial undertaking for DGOMs, but one that may be required to understand fully the implications of environmental change for its dynamics. This will be the subject of future research.

Seasonal forcings, at either the phytoplankton or zooplankton time scales, were shown to 
result in loss of stable dynamics in almost all cases (cf Figures 8, 9 and 10 with figures 4, 5 and 6). Such loss of stability, as suggested in recent empirical results [31], reveals mixotrophs as the dynamic disruptors of marine plankton ecosystems.

Further, Figure 11 suggests that even dynamical analyses will need to be implemented with great care, as the differences in the models may intermittent and subtle, and depend critically upon other assumptions made in the model. The heuristic that might have been inferred from the analyses of the $N P_{1} P_{2} Z$ model of Moroz et al [23], that models in which the $Z$ functioned as a generalist grazer were "more stable" than models in which it functioned as a specialist grazer, is weakened if $P_{2}$ fuels even a minor part of its growth from mixotrophic activities (i.e. $\mu_{2}=1.15$ vs $\phi_{3}=0.07$ ). If $N=0.5=P, M$ would obtain only $1 \%$ of its growth from grazing on $P$.

\section{Conclusion}

The "take-home" message from this analysis is that ecosystem models that are to be used in predictive scenarios, such as plankton models that are being developed for climate change predictions, require comprehensive analyses of both their equilibrium and dynamical properties. These may well be tightly coupled, with pinball dynamics that appear relatively common in these models requiring a synthesis of a complete equilibrium analysis (i.e. of all critical points) and numerical examination of the dynamics. This example has demonstrated that profound changes may be induced by very subtle changes in trophic interactions - we have allowed the mixotroph a maximum growth rate from consuming phytoplankton of only $0.69 \%$ of its maximum growth rate from photosynthesis (0.0074 vs 1.075). Further, changes in dynamical behaviour also depend on choices made elsewhere in the model, in this case the choice whether the zooplankton is a specialist or generalist grazer determines whether the level of mixotrophy affects the dynamics or not. Finally, external forcings such as seasonal variations further confound the dynamics, with effectively the same dynamics evident in different systems dramatically diverging before returning to effectively similar dynamics. Our conclusion then echos Thingstad et al's [27] observation that the examination of equilibria of simple models was a necessary preliminary to developing a deeper understanding of the dynamic behaviour of more complex/realistic models. The results of the analysis of this simple three-population model suggest that that deeper understanding will not come easily, and that extended sophisticated analyses of plankton models destined for inclusion in climate models is a 
fundamental component of their development and deployment. 


\section{References}

[1] Aumont, O., Bopp, L., 2006. Globalizing results from ocean in situ iron fertilization studies. Global Bio-geochemical Cycles 20, GB2017.

[2] Berge, T., Chakraborty, S., Hansen, P.J., Andersen, K.H., 2017. Modeling succession of key resource-harvesting traits of mixotrophic plankton. The ISME Journal 11:212-223.

[3] G. Billen and S. Becquevort. Phytoplankton: bacteria relationships in the Antarctic marine ecosystem. Pol.Res, 10, 245-253, 1991.

[4] Buitenhuis, E.T., Rivkin, R.B., Sailley, S., Le Quere, C., 2010. Bio-geochemical fluxes through microzooplankton. Global Bio-geochemical Cycles 24, GB4015.

[5] Caron, D.A., 2016. Mixotrophy stirs up our understanding of marine food webs. PNAS vol. 113(11): 2806-2808.

[6] Crane, K.W., Grover, J.P., 2010. Coexistence of mixotrophs, autotrophs, and heterotrophs in planktonic microbial communities. J. Theor Biol 262: 517-527.

[7] Cropp, R.A. and Norbury, J., 2009. Simple predator-prey interactions control dynamics in a plankton foodweb model. Ecol. Model. 220: 1552-65.

[8] Cropp, R.A., Moroz, I.M., Norbury, J., 2017. The role of grazer predation strategies in the dynamics of consumer-resource based ecological models. J. Sea Research vol. 125, 34-46.

[9] T. Fenchel. Ecology of heterotrophic microflagellates. IV. Quantitative occurrence and importance as bacterial consumers. Mar. Ecol. Progress Ser., 9, 35-42, 1982.

[10] Flynn K.J., Mitra A., 2009. Building the "perfect beast": modelling mixotrophic plankton. J Plank Res Vol 31(9): 965-992.

[11] Flynn K.J., Stoecker D.K., Mitra A., Raven J.A., Glibert P.M., Hansen P.J., Grane?li E., Burkholder J.M., 2013. Misuse of the phytoplankton? zooplankton dichotomy: the need to assign organisms as mixotrophs within plankton functional types. J. Plankton Res. 35(1): 3-11.

[12] Franks, P.J.S., 2002. NPZ models of plankton dynamics: their construction, coupling to physics, and application. J. Oceanogr. 58:379-387.

[13] A.J. Gabric, P.A. Matrai and M. Vernet. Modelling the production and cycling of dimethylsulphide during the vernal bloom in the Barents Sea. Tellus, 51B, 919-937, 1999.

[14] A.J. Gabric, P.H. Whetton and R.A. Cropp. Dimethylsulphide production in the sub-Antarctic southern ocean under enhanced greenhouse conditions. Tellus, 53, 273-287, 2001. 
[15] Ghyoot, C., Flynn, K.J., Mitra, A., Lancelot, C., Gypens, N., 2017. Modeling Plankton Mixotrophy: A Mechanistic Model Consistent with the Shuter-Type Bio-chemical Approach. Front. Ecol. Evol. 5:78. doi: 10.3389/fevo.2017.00078.

[16] B. Hansen, S. Christiansen and G. Pedersen. Plankton dynamics in the marginal ice zone of the central Barents Sea during spring: carbon flow and the structure of the grazer food chain. Polar Biol., 16, 115-128, 1996.

[17] Hartmann, M., Grob, C., Tarran, G.A., Martin, A.P., Burkill, P.H., Scanlan, D.J., Zubkov, M.V., 2012. Mixotrophic basis of Atlantic oligotrophic ecosystems. PNAS vol. 109(15): 5756-5760.

[18] Holling, C. S., 1965. The functional response of predators to prey density and its role in mimicry and population regulation. Memoirs of the Entomological Society of Canada, 45: $5-60$.

[19] G. Muller-Niklas and G.J. Herndl. Dynamics of bacterio-plankton during a phytoplankton bloom in the high Arctic waters of the Franz-Joseph Land archipelago. Aquat. Microb. Ecol., 11, 111-118, 1996.

[20] May, R.M. and Leonard, W.J., 1975. Nonlinear aspects of competition between three species. SIAM J. Appl. Math. 29: 243-53.

[21] Mitra A, Flynn K.J., Burkholder J.M., Berge T., Calbet A., Raven J.A., Granéli E., Glibert P.M., Hansen P.J., Stoecker D.K., Thingstad F., Tillmann U., Våge S., Wilken S., Zubkov M.V., 2014. The role of mixotrophic protists in the biological carbon pump. Biogeosciences 11(4):995-1005.

[22] C.L. Moloney, M.O. Bergh, J.G. Field and R.C. Newell. The effect of sedimentation and microbial nitrogen regeneration in a plankton community: a simulation investigation. $J$. Plankton Res., 8, 427-445, 1986.

[23] Moroz, I.M., Cropp, R.A., Norbury, J., 2016. Chaos in plankton models: foraging, strategy and seasonal forcing. Ecological Modelling vol. 332, 103-111.

[24] Sailley, S.F., Vogt, M., Doney, S.C., Aita, M.N., Bopp, L., Buitenhuis, E.T., Hashioka, T., Lima, I., Le Quere, C., Yamanaka, Y., 2013. Comparing food web structures and dynamics across a suite of global marine ecosystem models. Ecological Modelling 261-262: 43 ?57.

[25] D. Slagstad and K. Stole-Hansen. Dynamics of plankton growth in the Barents Sea. Polar Res., 10, 173-186, 1991. 
[26] Stoecker, D.K., Hansen, P.J., Caron, D.A., Mitra, A., 2016. Mixotrophy in the Marine Plankton. Annu. Rev. Mar. Sci. vol. 9:311-35.

[27] Thingstad, T.F., Havskum, H., Garde, K., Riemann, B., 1996. On the strategy of "eating your competitor": a mathematical analysis of algal mixotrophy. Ecology, Vol. 77(7): 2108-2118.

[28] Ullah, H., Nagelkerken, I., Goldenberg, S.U., Fordham, D.A., 2018. Climate change could drive marine food web collapse through altered trophic flows and cyanobacterial proliferation. PLOS Biology — https://doi.org/10.1371/journal.pbio.2003446.

[29] Vitousek, P. M., and Matson, P.A., 2012. Nutrient cycling and bio-geochemistry. in S. Levin, editor, The Princeton Guide to Ecology, Princeton University Press, New Jersey. Pages 330-339.

[30] Ward, B.A., Follows, M.J., 2015. Marine mixotrophy increases trophic transfer efficiency, mean organism size, and vertical carbon flux. PNAS vol. 113(11): 2958-2963.

[31] Wilken, S., Huisman, J., Naus-Wiezer, S., Van Donk, E., 2013. Mixotrophic organisms become more heterotrophic with rising temperature. Ecol. Lett. 16, 225-233. 


\section{Highlights}

- A two phytoplankton prey one zooplankton predator population model is investigated with Holling Type II grazing.

- One prey population remains an autotroph throughout, while the other prey population transitions from autotroph to mixotroph.

- Facultative and Obligative omnivory are compared.

- Mixotrophy is found to have a substantial effect on the nonlinear dynamical properties.

- Mixotrophy has a marked effect on the nonlinear dynamics of the Generalist $(\rho=1)$ case.

- The effects of seasonal forcing are also considered.

- Our analyses suggest the importance of understanding plankon models in their own rught, before incorporating them into climate models. 


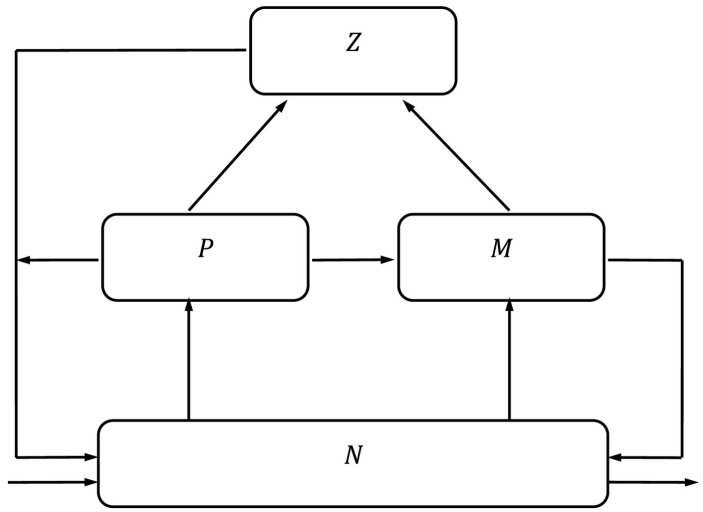

Figure 1 


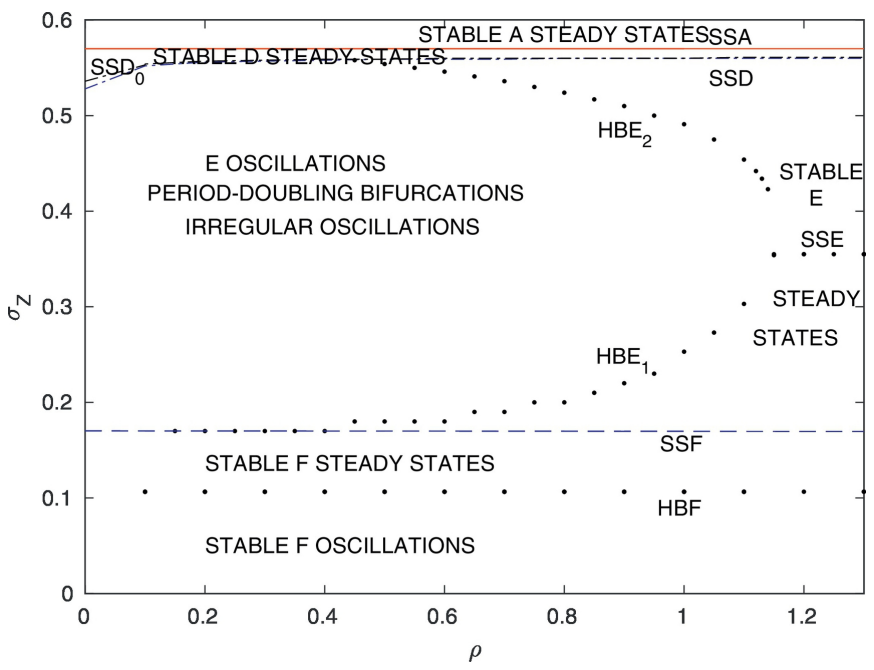

Figure 2 

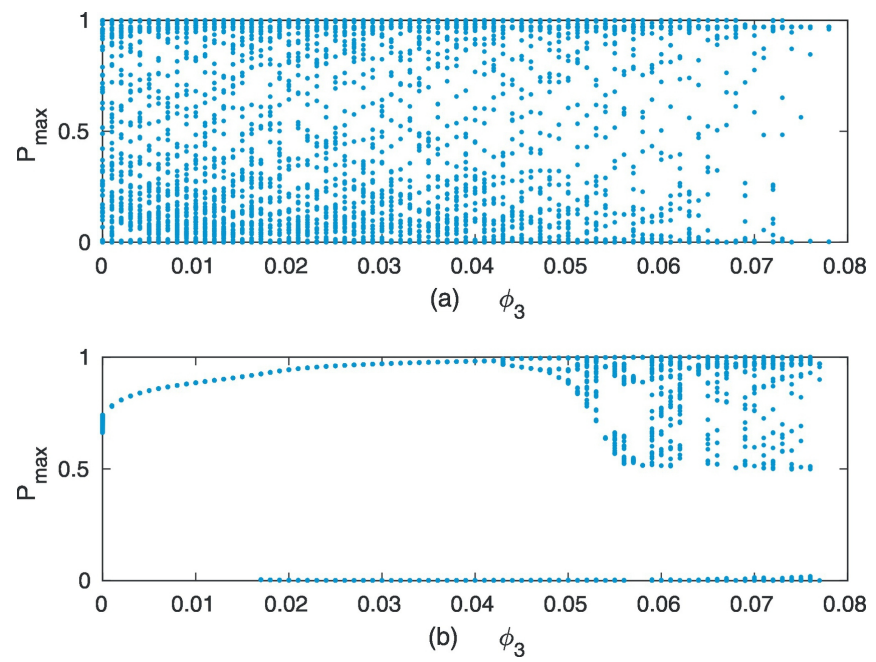

Figure 3 

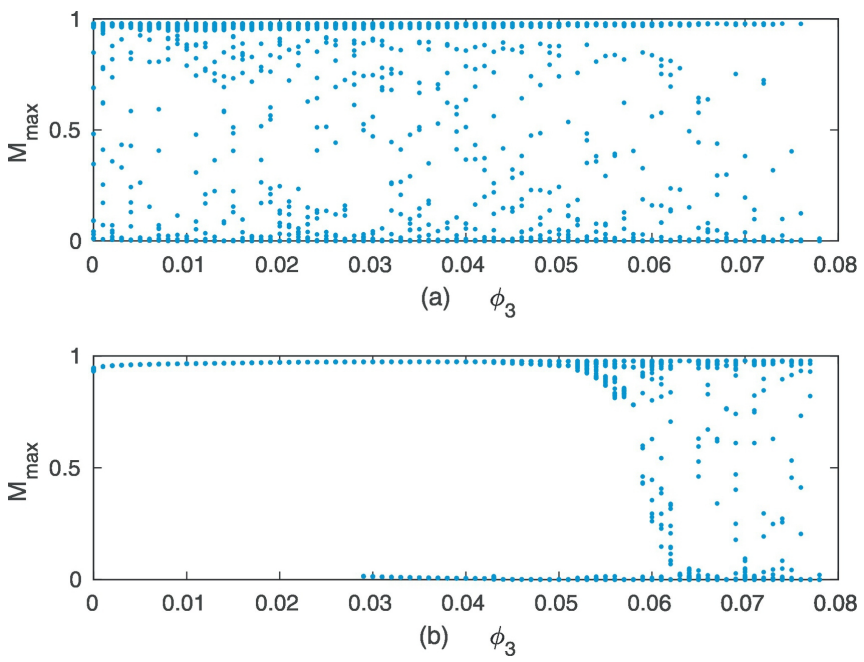

Figure 4 

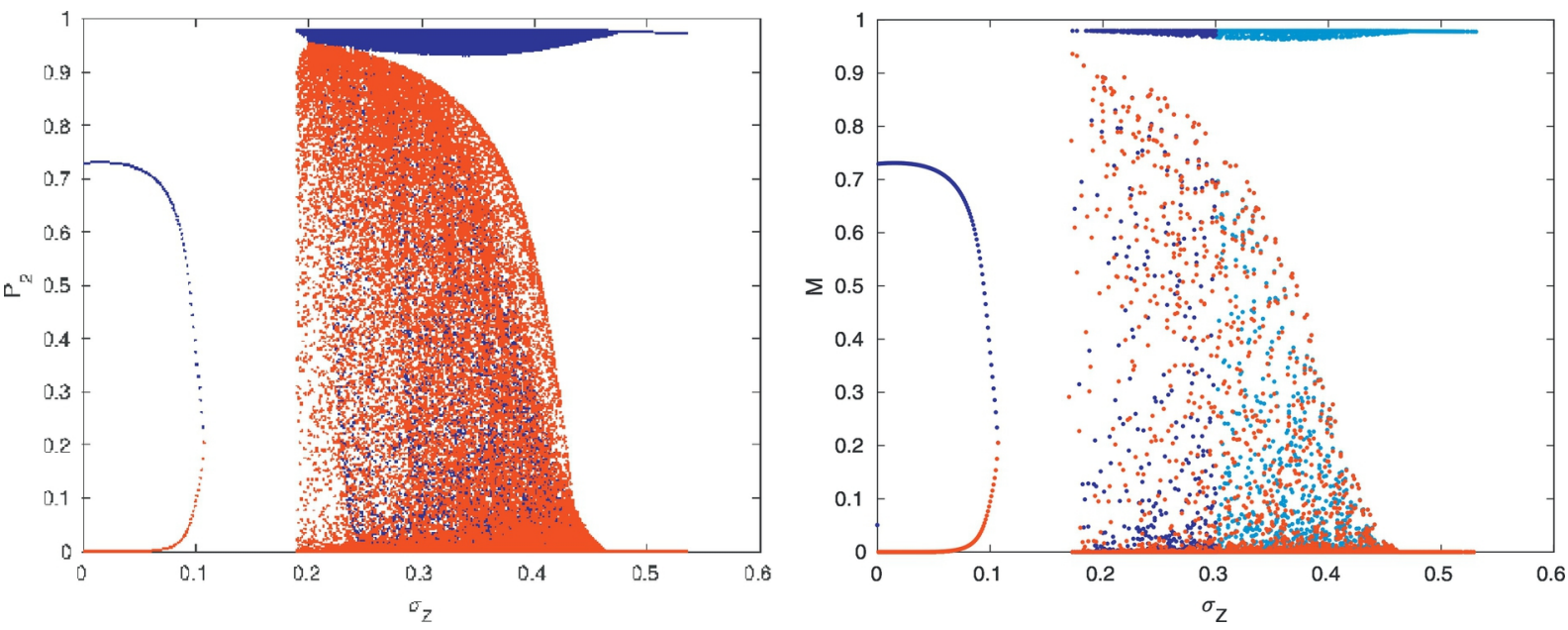

Figure 5 

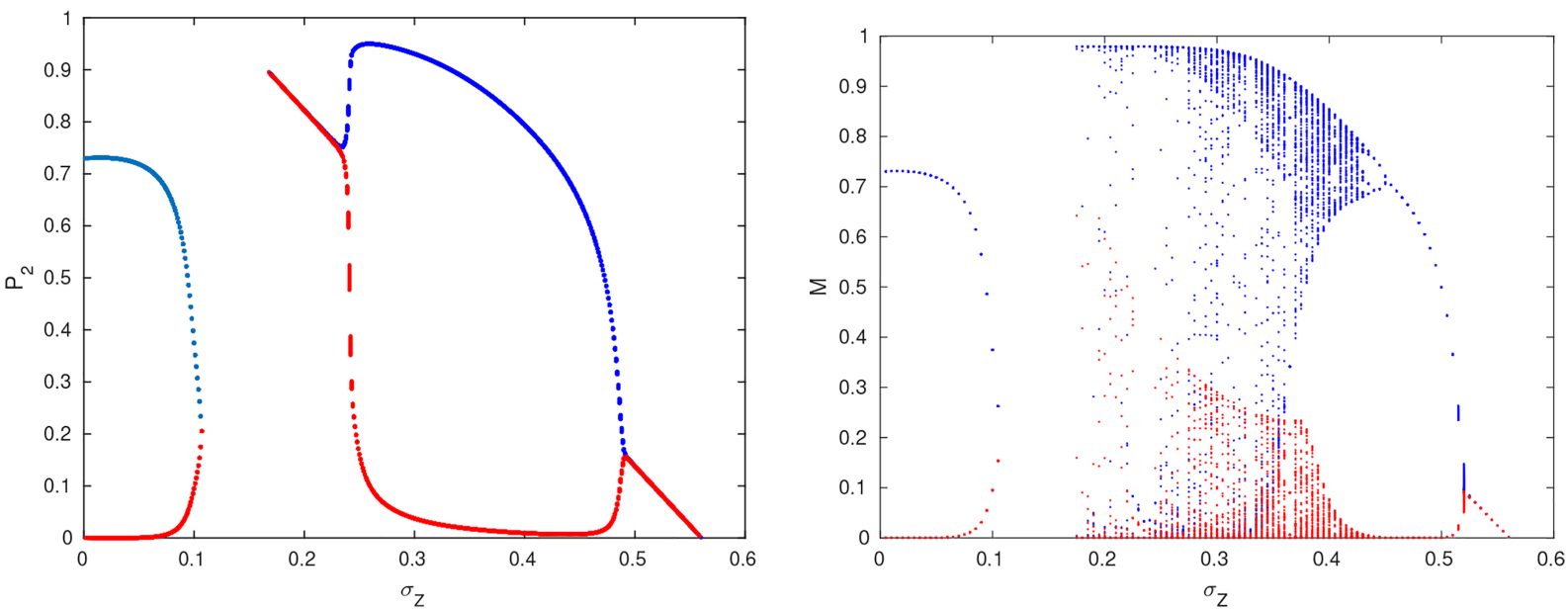

Figure 6 

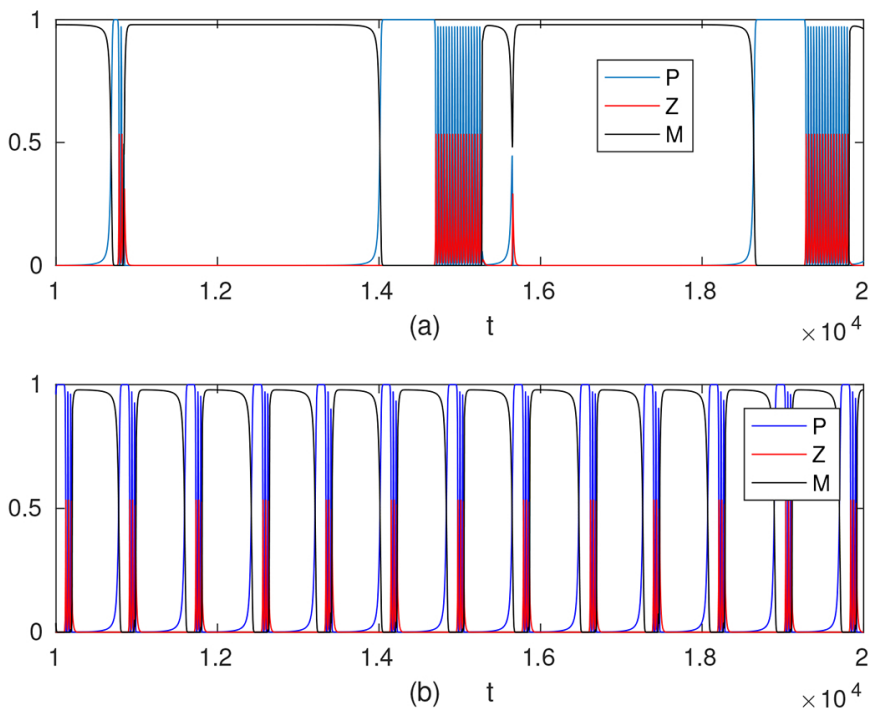

Figure 7 

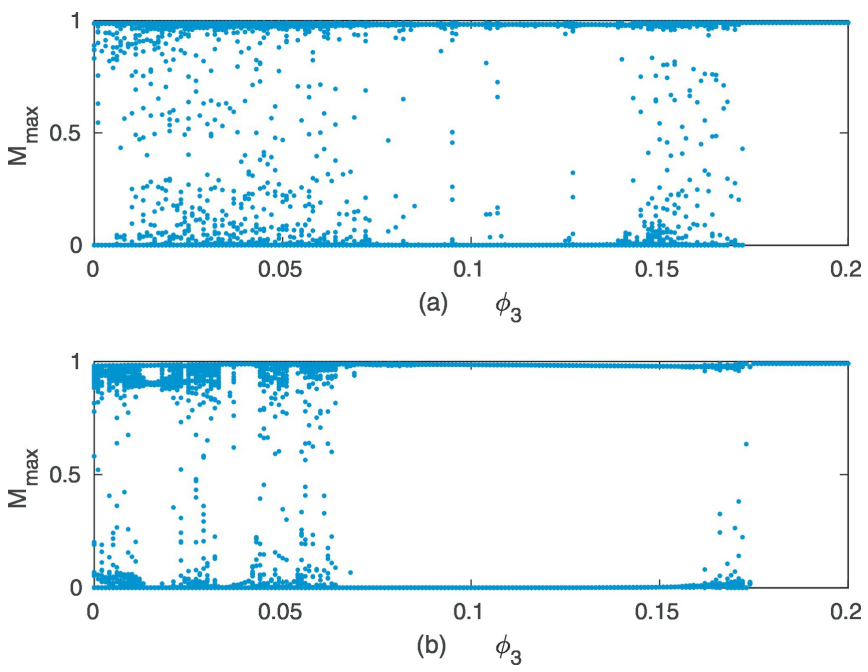

Figure 8 

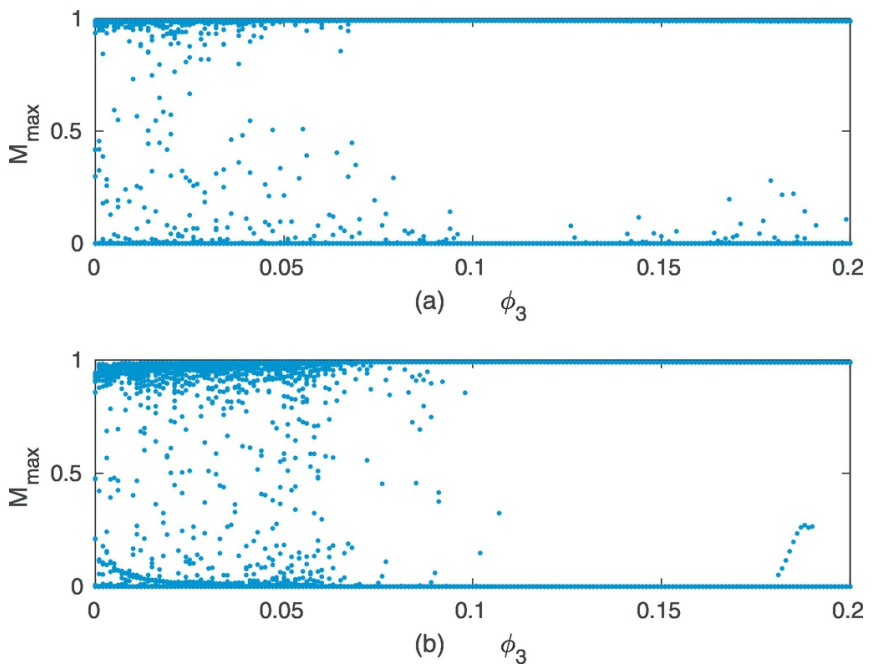

Figure 9 

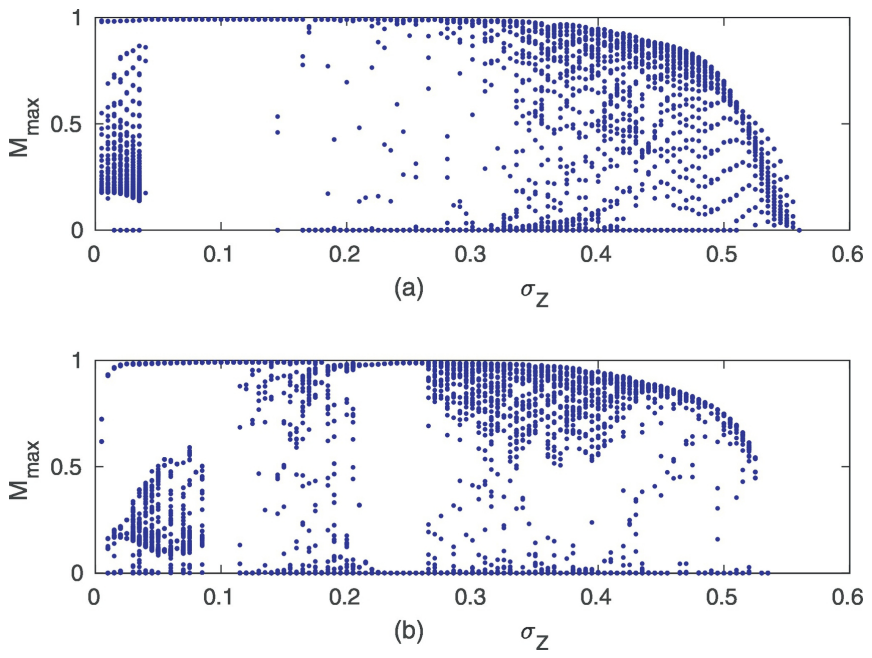

Figure 10 

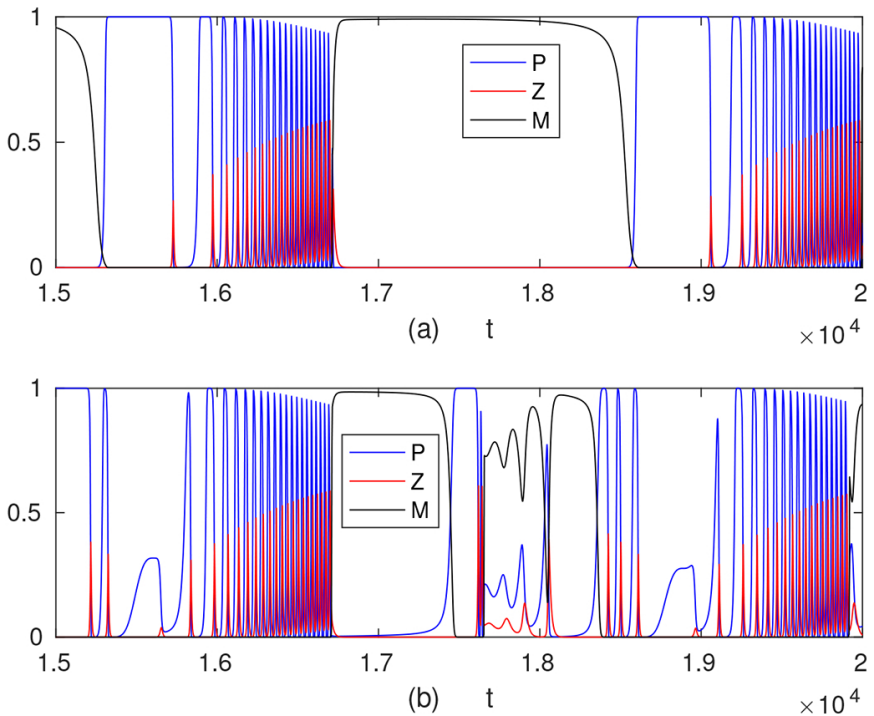

Figure 11 


\section{Accepted Manuscript}

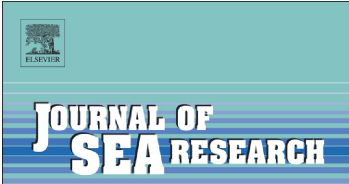

Mixotrophs: Dynamic disrupters of plankton systems?

Irene M. Moroz, Roger Cropp, John Norbury

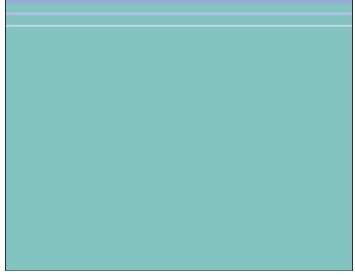

PII:

S1385-1101(18)30221-1

DOI: https://doi.org/10.1016/j.seares.2019.02.001

Reference: SEARES 1749

To appear in: Journal of Sea Research

Received date: 12 July 2018

Revised date: 28 January 2019

Accepted date: 4 February 2019

Please cite this article as: I.M. Moroz, R. Cropp and J. Norbury, Mixotrophs: Dynamic disrupters of plankton systems?, Journal of Sea Research, https://oi.org/10.1016/ j.seares.2019.02.001

This is a PDF file of an unedited manuscript that has been accepted for publication. As a service to our customers we are providing this early version of the manuscript. The manuscript will undergo copyediting, typesetting, and review of the resulting proof before it is published in its final form. Please note that during the production process errors may be discovered which could affect the content, and all legal disclaimers that apply to the journal pertain. 